\title{
Laterally Nanostructured Vesicles, Polygonal Sheets and Anisotropically \\ Patched Micelles from Solution-State Self-Assembly of Miktoarm Star \\ Quaterpolymers: A Simulation Study
}

Jiaping Wu, Zheng Wang, Yuhua Yin, Run Jiang, Baohui Li*

School of Physics, Key Laboratory of Functional Polymer Materials of Ministry of Education, Nankai University, and Collaborative Innovation Center of Chemical Science and Engineering (Tianjin), Tianjin, 300071, China

* To whom correspondence should be addressed. E-mail: baohui@ nankai.edu.cn 

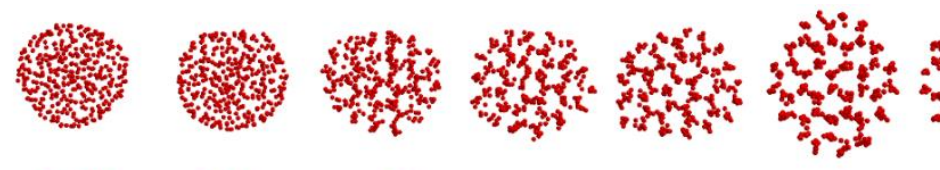

b
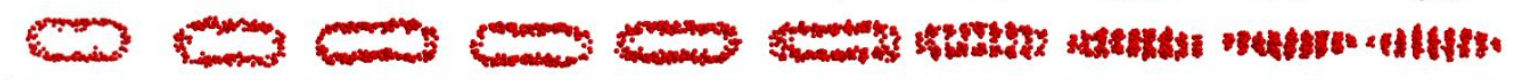

0

0.2

0.4

$0.6 \quad 0.8$

$1.0 \quad 1.5$

2.0

2.5

$4.0 \quad \alpha$

Figure S1. The distribution of the junction points D as a function of $\alpha$ for the nanosheets shown in Figure 1, where plots in (a) and (b) are viewed in two perpendicular directions for each morphology, and those in (b) is a thin slice in the cross-sectional view.
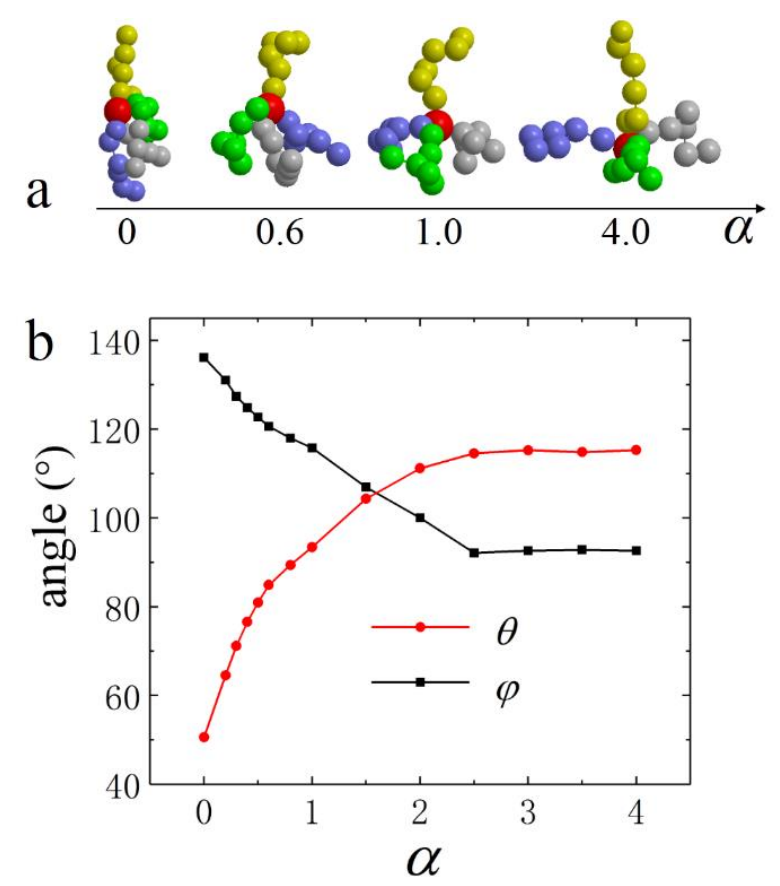

Figure S2. (a) Typical chain configuration and (b) the average angle $\theta$ and $\varphi$ as a function of $\alpha$ for the nanosheets shown in Figure 1. $\theta=\left(\theta_{\mathrm{AB}}+\theta_{\mathrm{BC}}+\theta_{\mathrm{AC}}\right) / 3$ and $\varphi=\left(\varphi_{\mathrm{AP}}+\varphi_{\mathrm{BP}}+\varphi_{\mathrm{CP}}\right) / 3$ represent the average angle between the different solvophobic arms, and between the solvophobic arm and the solvophilic arm, respectively, where $\theta_{\mathrm{IJ}}$ denotes the angle between vectors from the junction point $\mathrm{D}$ 
to the centers of mass of the $\mathrm{I}$ arm and the $\mathrm{J}$ arm, with $\mathrm{I}, \mathrm{J}=\mathrm{A}, \mathrm{B}, \mathrm{C}$, and $\mathrm{P}$. The angles $\theta$ and $\varphi$ reach their respectively stable values of $\sim 120^{\circ}$ and $\sim 90^{\circ}$ at $\alpha \geq 2.5$, indicating that the three solvophobic arms split a plane equally, while the solvophilic arm is perpendicular to that plane.

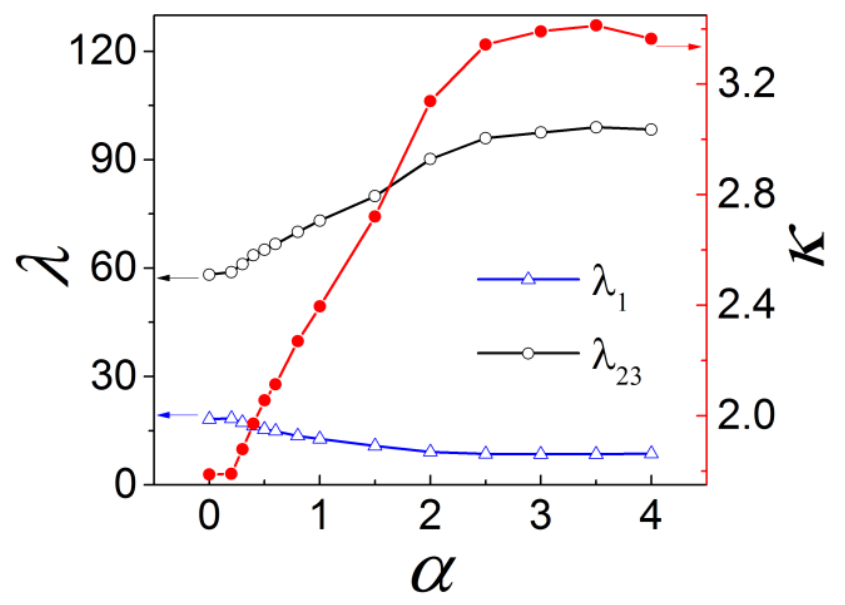

Figure S3. $\lambda_{1}, \lambda_{23}$ and the aspect ratio $\kappa$ as a function of $\alpha$, where $\lambda_{1}, \lambda_{2}, \lambda_{3}$ (with $\lambda_{1} \leq \lambda_{2} \leq \lambda_{3}$ ) are the three eigenvalues of the moment of inertia tensor for the nanosheets shown in Figure $1, \lambda_{23} \equiv$ $\left(\lambda_{2}+\lambda_{3}\right) / 2$ and the aspect ratio $\kappa=\left(\lambda_{23} / \lambda_{1}\right)^{1 / 2}$. The case of $\lambda_{1}=\lambda_{2}=\lambda_{3}(\kappa=1)$ corresponds to a standard sphere, while the case of $\lambda_{1}=\lambda_{2}<<\lambda_{3}(\kappa>1)$ to a rod, and that of $\lambda_{1}<<\lambda_{2}=\lambda_{3}(\kappa>1)$ to a standard disk. The Figure shows that with the increase of $\alpha$, the radial size of the nanosheets (characterized by $\lambda_{23}$ ) increases while its thickness (characterized by $\lambda_{1}$ ) decreases. This result is consistent with the observations in Figure 1(b, c). 

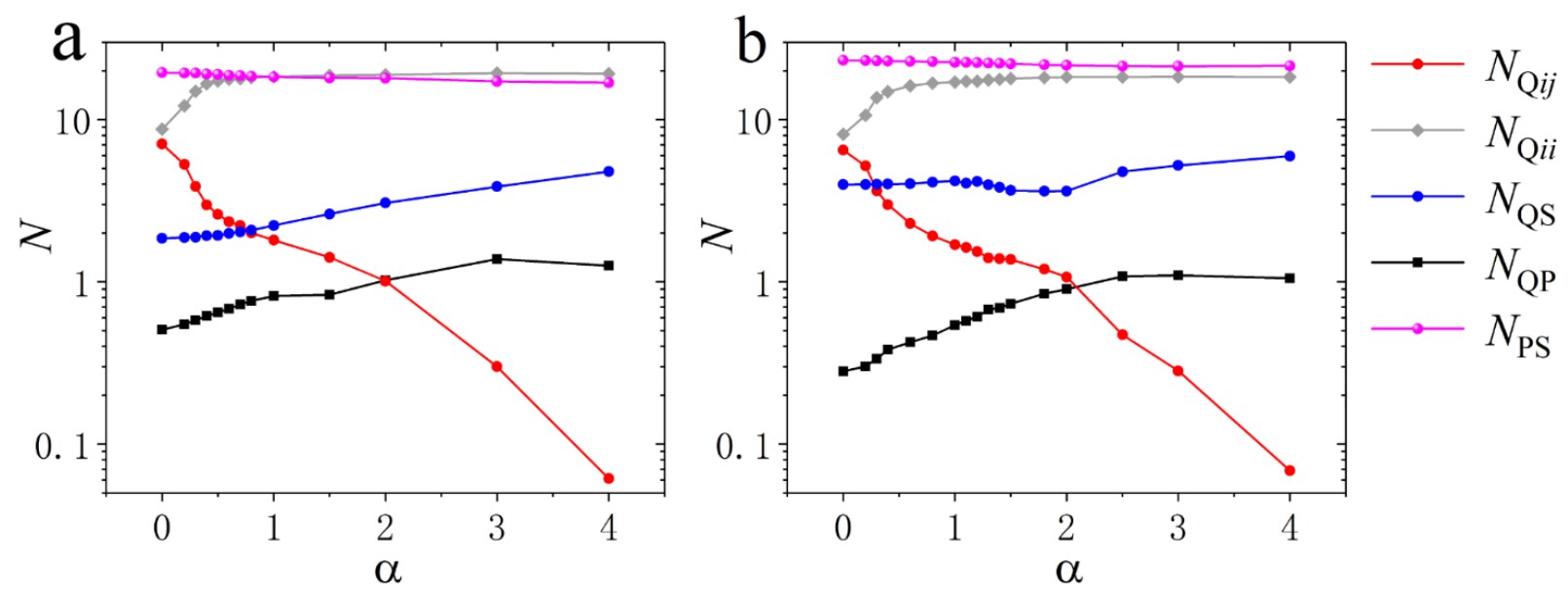

Figure S4. The average contact numbers $N_{\mathrm{Q}_{i j}}, N_{\mathrm{Q}_{i i}}, N_{\mathrm{QS}}, N_{\mathrm{QP}}, N_{\mathrm{PS}}$ as a function of $\alpha$ for the morphologies shown (a) in Figure $3\left(N_{\mathrm{P}}=4\right)$ and $(\mathrm{b})$ in Figure $4\left(N_{\mathrm{P}}=10\right)$. 


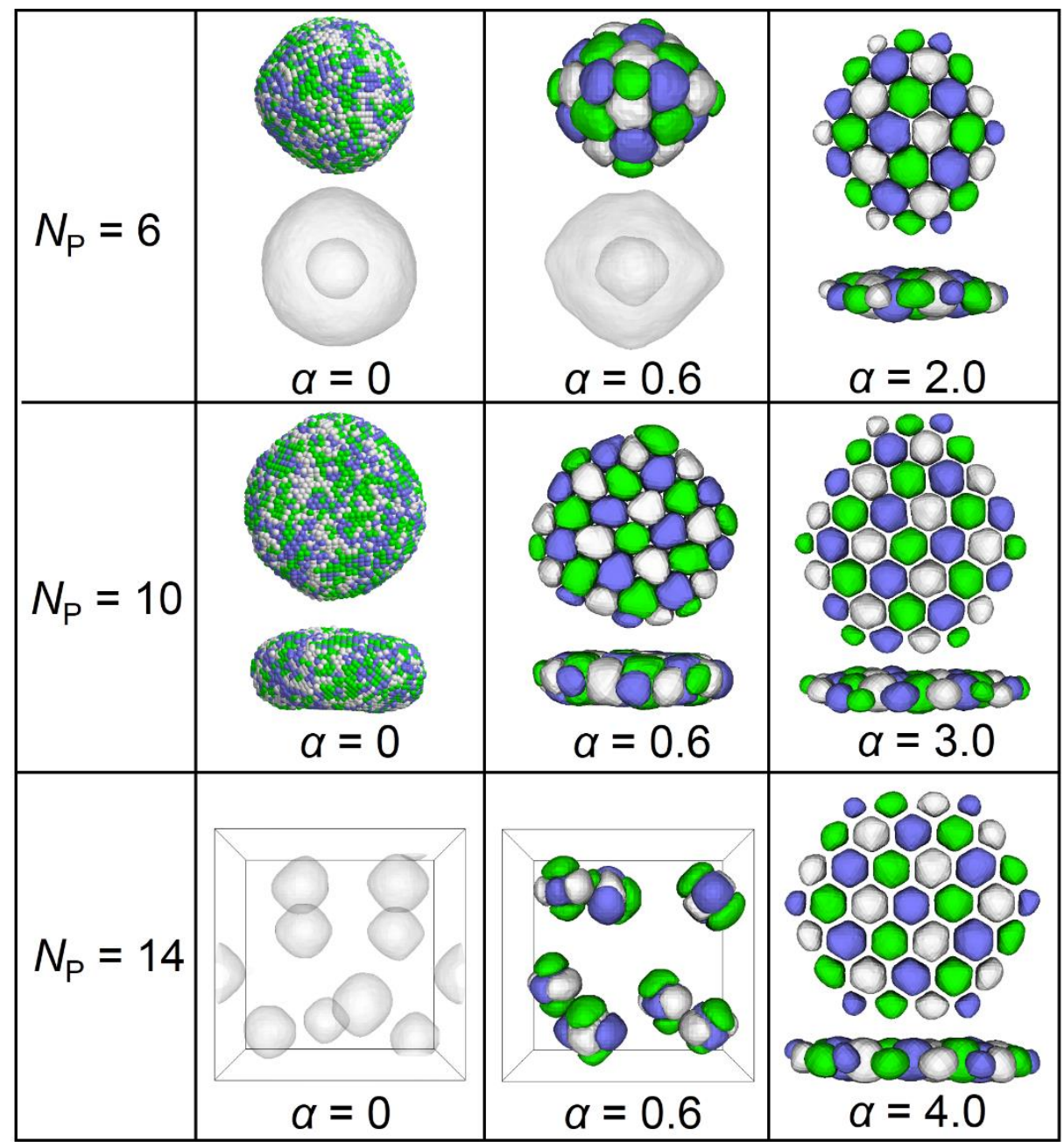

Figure S5. Typical snapshots of the self-assembled morphologies as a function of $N_{\mathrm{P}}$ and $\alpha$ at $N_{\mathrm{Q}}=12$, $\Phi=0.06, \varepsilon_{\mathrm{AS}}=\varepsilon_{\mathrm{BS}}=\varepsilon_{\mathrm{CS}}=2.0$ and $V=72^{3}$ with only the solvophobic (ABC) domains are shown in isosurface contour plots except the upper plot at $\alpha=0$ and $N_{\mathrm{P}}=6$ and the plots at $\alpha=0$ and $N_{\mathrm{P}}=10$. All nanosheets are viewed in two perpendicular directions. The A, B and C domains in vesicles are shown in different colors and in one color, and those in spherical micelles are shown in different colors at $\alpha=0.6$ and in one color at $\alpha=0$. The color scheme is the same as that used in Figure 1 . 


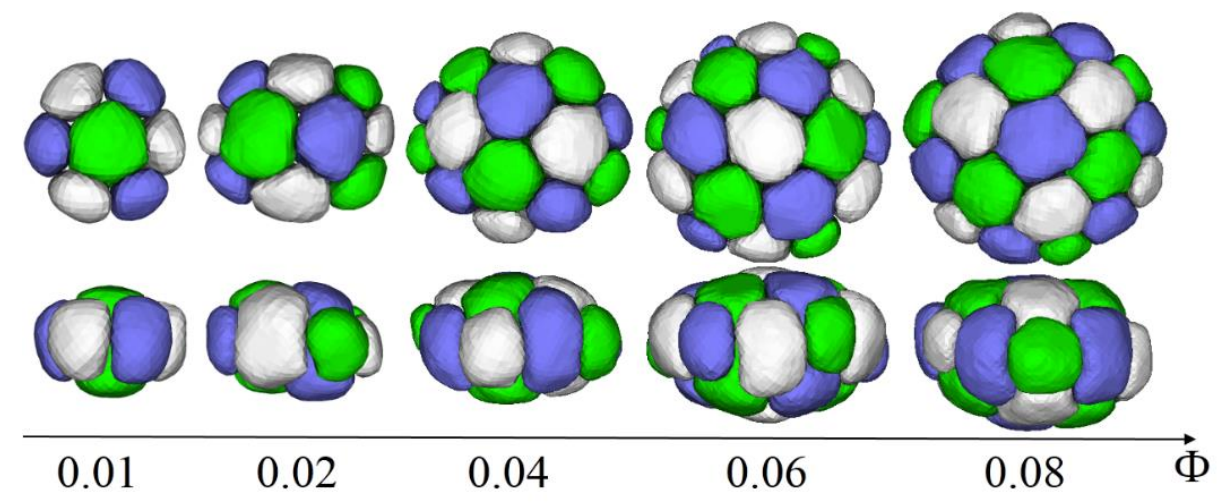

Figure S6. Typical snapshots of the self-assembled morphologies as a function of the segment concentration $\Phi$ shown in isosurface contour of the solvophobic domains $(\mathrm{ABC})$ where $N_{\mathrm{P}}=2, N_{\mathrm{Q}}=24$, $\alpha=2 / 3, \varepsilon_{\mathrm{AS}}=\varepsilon_{\mathrm{BS}}=\varepsilon_{\mathrm{CS}}=3.0$ and $V=80^{3}$. Plots in the two rows are viewed in two perpendicular directions. The color scheme is the same as that used in Figure 1.

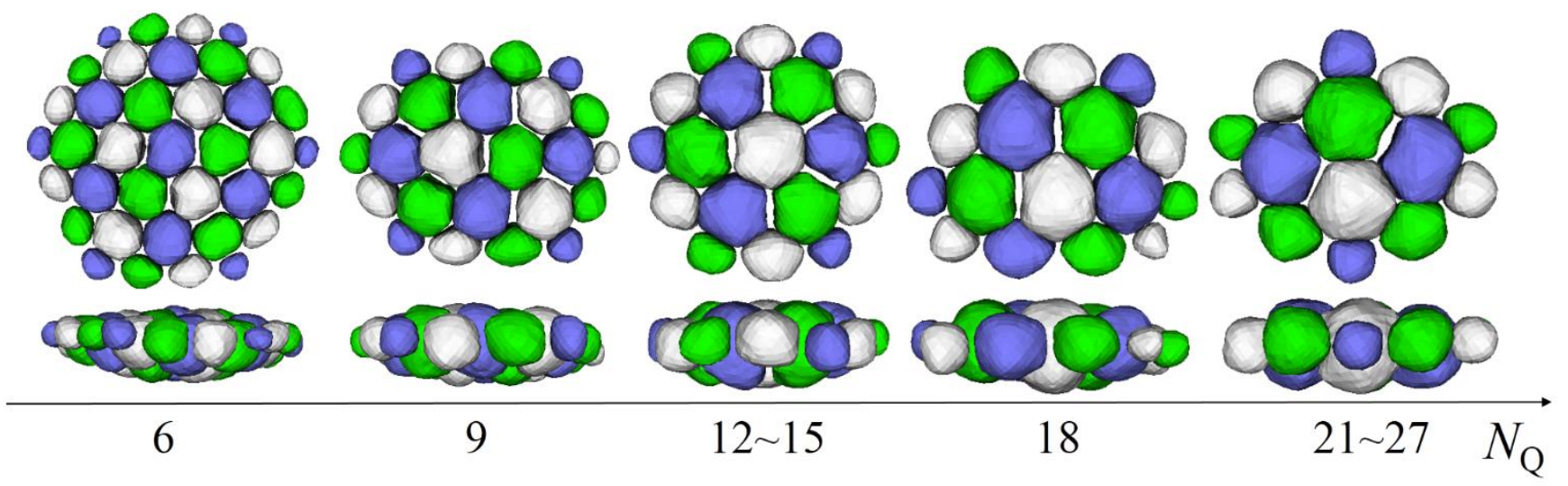

Figure S7. Typical snapshots of self-assembled morphologies as a function of the solvophobic arm length $N_{\mathrm{Q}}$ when $N_{\mathrm{P}}=2, \alpha=2.0, \varepsilon_{\mathrm{AS}}=\varepsilon_{\mathrm{BS}}=\varepsilon_{\mathrm{CS}}=1.0, \Phi=0.07$, and $V=60^{3}$. The plot formats and the color scheme are the same as those used in Figure S6 and Figure 1, respectively. Polygonal shape appears due to the large $\alpha$ value $(\alpha=2)$. 